\title{
Super resolution DOA estimation with incomplete single snapshot data via matrix completion and compress sensing
}

\author{
Dong Zhang, ${ }^{1, *}$, Qin Zhang ${ }^{1}$, Baojun Song ${ }^{1}$, Guimei Zheng ${ }^{1}$, and Yongshun Zhang ${ }^{1}$ \\ ${ }^{1}$ Air and Missile Defense College, Air Force Engineering University, Xi'an 710051, China
}

\begin{abstract}
A method of super resolution DOA estimation with incomplete single snapshot data via matrix completion (MC) and compress sensing (CS) is presented. First, the incomplete single snapshot data is reshaped into a low-rank Hankel matrix form and the complete data can be reconstructed through matrix completion. Then, super resolution DOA estimation can be got through CS algorithm using these constructed complete data. Numerical simulations demonstrate that this method can get high accuracy DOA estimation with less number of array elements.
\end{abstract}

\section{Introduction}

DOA is a very important parameter in Radar system and its estimation accuracy will seriously affect the performance of Radar system. DOA estimation of maneuvering targets is usually difficult because the available snapshot number is usually rare. So the DOA estimation with short snapshots is receiving more and more attention and many literature devote to it [1]. More recently, DOA estimation based on CS (CS-DOA) becomes a hot field because of its advantage in fully exploiting the inherent sparseness of the DOA [2] and reducing the observation.

As we know, matrix completion is a novel technique which emerges on the heels of compress sensing[3]. It can recover a low-rank matrix which is incomplete and perhaps even corrupted. So it has the potential of reducing the array number. In this paper, we proposed a novel DOA estimation method which combines matrix completion with compress sensing under the condition of incomplete single snapshot. First, we reshape the incomplete single snapshot data into a low-rank Hankel matrix form and we can get the complete data through matrix completion. Then, we can get super resolution DOA estimation via CS algorithm using the reconstructed complete data. The matrix completion theory ensures that we can get the accurate complete data with the array number reducing to a certain extent. The compress sensing theory ensures that we can get super resolution estimation with the reconstructed complete data. Thus, this method can obtain super resolution DOA estimation with less array number.

\section{Signal model}

Assume there is a uniform linear array (ULA)which has $N$ receivers. The interval between array elements is $d$.The signal of targets are incoming from $M$ different direction which are $\theta_{i}, i=1,2, \ldots, M$

And $f_{i}=d \cos \left(\theta_{i}\right) / \lambda$ are the normalized DOA. In the rest of the paper, we will directly refer to it as DOA for simplicity.

Then the complete output of the receive array with single snapshot can be expressed as

$$
\boldsymbol{x}_{\boldsymbol{C}}=\boldsymbol{A}(\theta) \boldsymbol{s}+\boldsymbol{w}=\sum_{i=0}^{M-1} \boldsymbol{a}\left(\theta_{i}\right) s_{i}+\boldsymbol{w}
$$

where $\boldsymbol{x}_{C}=\left[x_{0}, x_{1}, \ldots, x_{N-1}\right]^{T}$ denotes the received complete data of $N$ receivers with single snapshot. $\boldsymbol{w}=\left[w_{0}, w_{1}, \ldots, w_{N-1}\right]^{T}$ refers to the noise vector. $\boldsymbol{s}=\left[s_{0}, s_{1}, \ldots, S_{M-1}\right]^{T}$ denotes incident signal of $M \quad$ targets. $\quad \boldsymbol{a}\left(\theta_{i}\right)=\left[a_{0}\left(\theta_{i}\right), a_{1}\left(\theta_{i}\right), \ldots, a_{N-1}\left(\theta_{i}\right)\right]^{T}$ $=\left[1, \ldots, \exp \left(\mathrm{j}(N-1) 2 \pi f_{i}\right)\right]^{T}$ are receive steering vectors corresponding to the DOA of $i^{\text {th }}$ target and $\boldsymbol{A}(\theta)=\left[\boldsymbol{a}\left(\theta_{0}\right), \boldsymbol{a}\left(\theta_{1}\right), \ldots, \boldsymbol{a}\left(\theta_{M-1}\right)\right]$.

Discretize the range of DOA to $N_{L}>>N$ resolution grids. The CS theory ensures that, under certain conditions, we can get the super resolution of DOA by solving a sparse reconstruction problem.

But we want to reduce the number of array elements as more as possible. For example, we only want to use $N_{S}$ array elements. And we assume that they are randomly picked from $N$ array elements. If we continue use the CS method, the performance will decrease for the reduction of observation.

Note that the CS method theory utilizes the inherent sparseness of targets to get high resolution. But, in addition to sparsity, the received signals have the property of low rank. It ensures that we can use the partial data (we determine it as incomplete data)to recovery the full data(we determine it as complete data)

\footnotetext{
* Corresponding author: zhangdongtougao@163.com
} 
via matrix completion theory. So the combination of matrix completion with compress sensing will bring the advantage of get higher resolution estimation of DOA with less array elements.

\section{MC-CS algorithm}

According to Equation (1), the received complete data of $N$ receivers with single snapshot is $\boldsymbol{x}_{\boldsymbol{C}}=\left[x_{1}, x_{2}, \ldots, x_{N}\right]^{T}$ and the output of the receive array element $n(n=0,1, \ldots, N-1)$ can be expressed as

$$
x_{n}=\sum_{i=0}^{M-1} a_{n}\left(\theta_{i}\right) s_{i}+w_{n}, n=0,1, \ldots, N-1
$$

where $a_{n}\left(\theta_{i}\right)=\exp \left[\mathrm{j} n 2 \pi f_{i}\right]$.Then we randomly pick $N_{S}$ array elements from the ULA and $N_{S}<N$. The incomplete

data is $\boldsymbol{x}_{\boldsymbol{I C}}=\left[x_{1}, x_{2}, \ldots, x_{N_{S}}\right]^{T}$.Let $\boldsymbol{x}_{\boldsymbol{Z P}}$ represents the zeropadding result of incomplete data $\boldsymbol{x}_{I C}$. Then we can regard $\boldsymbol{x}_{\boldsymbol{Z} \boldsymbol{P}}$ as an incomplete measurement of $\boldsymbol{x}_{\boldsymbol{C}}$

$$
\left[\boldsymbol{x}_{Z P}\right]_{l}=\left[\boldsymbol{x}_{\boldsymbol{C}}\right]_{l}, l \in \Omega
$$

where $[\cdot]_{l}$ stands for the $l$ th item of the vector. $\Omega$ denotes a subset of $[0: N-1]$ and it represents the array elements which are random picked. Another form of Equation (5) is $\boldsymbol{x}_{\boldsymbol{Z P}}=\mathrm{P}_{\Omega}\left(\boldsymbol{x}_{\boldsymbol{C}}\right)$. Where $\mathrm{P}_{\Omega}(\cdot)$ stands for the operation of randomly selection.

Based on complete data $\boldsymbol{x}_{C}=\left[x_{1}, x_{2}, \ldots, x_{N}\right]^{T}$, a Hankel matrix can be constructed as follows

$$
\boldsymbol{H a n k}\left(\boldsymbol{x}_{\boldsymbol{C}}\right)=\left[\begin{array}{cccc}
x_{0} & x_{1} & \ldots & x_{N-d} \\
x_{1} & x_{2} & \ldots & x_{N-d+1} \\
\vdots & \vdots & \vdots & \vdots \\
x_{d-1} & x_{d} & \ldots & x_{N-1}
\end{array}\right]
$$

where $d$ is a positive integer which is often set as $d=\frac{N}{2}$ or $d=\frac{N+1}{2}$.According to Equation (2), we can decompose the Hankel matrix into the following form

$$
\operatorname{Hank}\left(x_{C}\right)=U \Sigma V
$$

where $\quad \boldsymbol{U}=\left[\begin{array}{cccc}a_{0}\left(\theta_{0}\right) & a_{0}\left(\theta_{1}\right) & \cdots & a_{0}\left(\theta_{M-1}\right) \\ a_{1}\left(\theta_{0}\right) & a_{1}\left(\theta_{1}\right) & \cdots & a_{1}\left(\theta_{M-1}\right) \\ \vdots & \vdots & \vdots & \vdots \\ a_{d-1}\left(\theta_{0}\right) & a_{d-1}\left(\theta_{1}\right) & \cdots & a_{d-1}\left(\theta_{M-1}\right)\end{array}\right]$

$\boldsymbol{V}=\left[\begin{array}{cccc}a_{0}\left(\theta_{0}\right) & a_{1}\left(\theta_{0}\right) & \cdots & a_{N-d}\left(\theta_{0}\right) \\ a_{0}\left(\theta_{1}\right) & a_{1}\left(\theta_{1}\right) & \cdots & a_{N-d}\left(\theta_{1}\right) \\ \vdots & \vdots & \vdots & \vdots \\ a_{0}\left(\theta_{M-1}\right) & a_{1}\left(\theta_{M-1}\right) & \cdots & a_{N-d}\left(\theta_{M-1}\right)\end{array}\right]$

and

$\Sigma=\operatorname{diag}\left(s_{0}, s_{1}, \ldots, s_{M-1}\right)$.

It obvious that $\operatorname{rank}\left[\boldsymbol{H a n k}\left(\boldsymbol{x}_{\boldsymbol{C}}\right)\right] \leq M$.

Then, according to the matrix completion theory, the estimation result $\tilde{\boldsymbol{x}}_{\boldsymbol{C}}$ of $\boldsymbol{x}_{\boldsymbol{C}}$ can be estimated through solving the problem as

$$
\min \left\|\operatorname{Hank}\left(\boldsymbol{x}_{\boldsymbol{C}}\right)\right\|_{*} \text {, s.t. } \mathrm{P}_{\Omega}\left(\boldsymbol{x}_{\boldsymbol{C}}-\boldsymbol{x}_{\boldsymbol{Z} \boldsymbol{P}}\right) \leq \varepsilon
$$

where \|\|$_{*}$ denotes the nuclear norm. This matrix completion problem can be solved by many algorithms, like the inexact augmented Lagrange multiplier algorithm [4] which possess high computational efficiency and adequate precision.

After get the estimation result $\tilde{\boldsymbol{x}}_{C}$, we can use the CS method to get super resolution DOA estimation. Discretizing the range of DOA to $N_{L}>N$ resolution grids, the estimation data can be expressed as

$$
\begin{aligned}
\tilde{\boldsymbol{x}}_{\boldsymbol{C}} & =\boldsymbol{\Phi}(\theta) \boldsymbol{s}_{\text {sparse }}+\boldsymbol{w} \\
& =\sum_{i=0}^{N_{L}} \boldsymbol{a}\left(\theta_{i}\right) s_{i}+\boldsymbol{w}
\end{aligned}
$$

where $\boldsymbol{\Phi}(\theta)=\left[\boldsymbol{a}\left(\theta_{0}\right), \boldsymbol{a}\left(\theta_{1}\right), \ldots, \boldsymbol{a}\left(\theta_{N_{L}-1}\right)\right]$ is the super resolution DOA dictionary, $\boldsymbol{s}_{\text {sparse }}=\left[s_{0}, s_{1}, \ldots, s_{N_{L}-1}\right]^{T}$ is the sparse vector whose non-zero elements' position represent the super resolution DOA estimation result. The CS theory shows that [5], if $\boldsymbol{A}_{\text {new }}(\theta)$ possess the restricted isometry property(RIP), then $\boldsymbol{s}_{\text {sparse }}$ will be reconstructed precisely according to $\tilde{\boldsymbol{x}}_{\boldsymbol{C}}$ via solving a optimization problem as

$$
\min \left\|\boldsymbol{s}_{\text {sparse }}\right\|_{1} \text { s.t. }\left\|\tilde{\boldsymbol{x}}_{C}-\boldsymbol{\Phi}(\theta) \boldsymbol{s}_{\text {sparse }}\right\|_{2} \leq \delta
$$

Among the reconstruction methods, OMP is usually seemed as the more suitable method for application because of its fast computation speed and satisfactory accuracy. So we solve Equation (8) by OMP algorithm. 


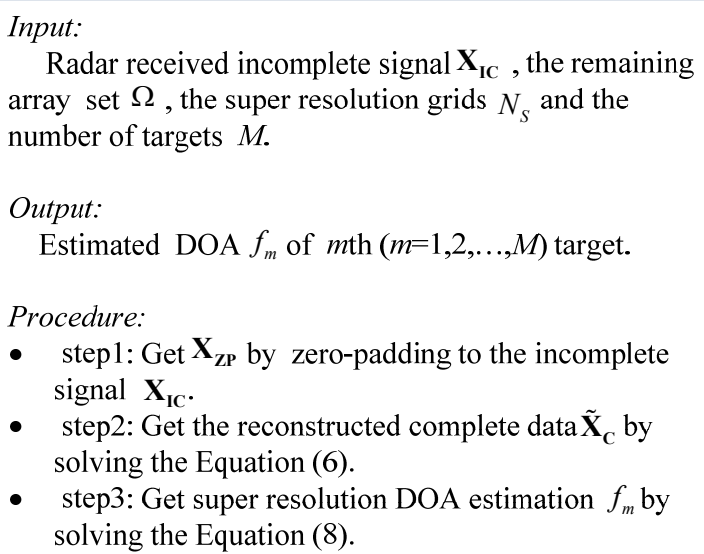

- step3: Get super resolution DOA estimation $f_{m}$ by solving the Equation (8).

\section{Figure 1: MC-CS algorithm}

This method is termed as the matrix completioncompress sensing (MC-CS) algorithm in this paper. Using this method, we can get high resolution estimation of DOA with less number of array elements. In summary, the MC-CS algorithm consists of three main steps is obtained which is given in Fig. 1.

\section{Simulation results}

of $m^{\text {th }}$ target are $f_{m}=0.2 m-0.66$. We made three kinds of simulation. In all the simulation, we set $N=50$ and $N_{L}=150$, i.e. the number of complete array and discretizing grids are 50 and 150 respectively. And all the simulation are conducted in the environment of additive white Gaussian noise.

For the first simulation, we set $\mathrm{SNR}=15 \mathrm{~dB}$. Then we randomly pick 38 array elements and the DOA estimation performance of the proposed MC-CS algorithm is shown in Fig. 2 which indicates that our method can obtain satisfactory estimation performance.

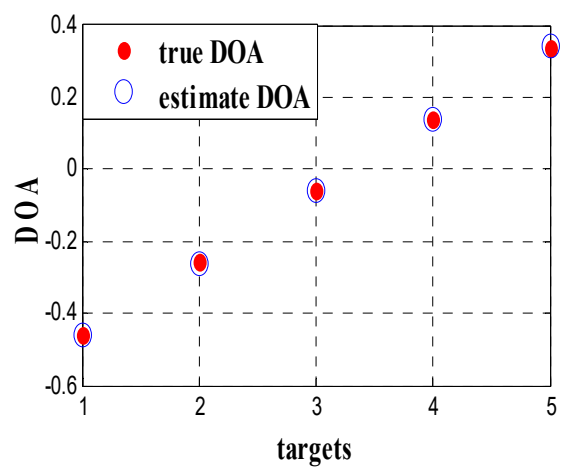

Fig.2 DOA estimation result of the proposed method

For the second simulation, we contrast the variation of average root-mean-square error (RMSE) of estimated DOA of all five targets with different SNR for the proposed MC-CS algorithms. The contrast test is the CS method using the incomplete data. The SNR varies from $0 \mathrm{~dB}$ to $30 \mathrm{~dB}$. 200 trails Monte Carlo simulations are performed for each given SNR. The other parameters are same with the first simulation. And the result is presented in Fig. 3 which indicates that the result of the proposed MC-CS algorithm is better than the CS method. Especially when $\mathrm{SNR}<-15 \mathrm{~dB}$, the proposed method is better than the CS which indicates that the proposed method have better performance in low SNR.

In the third simulation, we compare the RMSE of estimated DOA of all five targets with the picking number of array elements for CS method and the proposed MC-CS algorithm. The SNR is 15dB.Fig. 4 shows the comparison result with picking number from 27 to 47 .It obviously that the proposed MC-CS method is better than the CS method. We can see that with the decreasing of picking number, the CS method decrease significantly. While when the picking number is large than 41 , the estimation performance of MC-CS method is constant. It indicates that our method have a tolerant limit of the picking number. If the picking number is in the tolerant limit, our method will not be affect by the picking number. This property can help us to reduce the array elements.

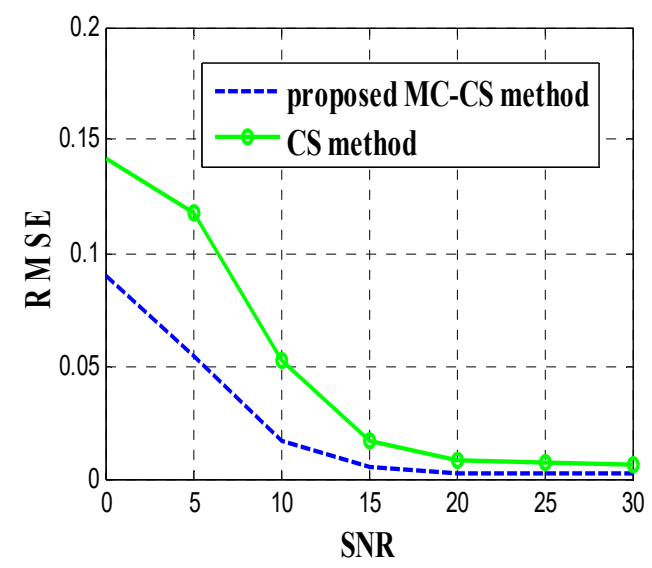

Fig. 3 Average RMSE of DOA estimation with different SNR

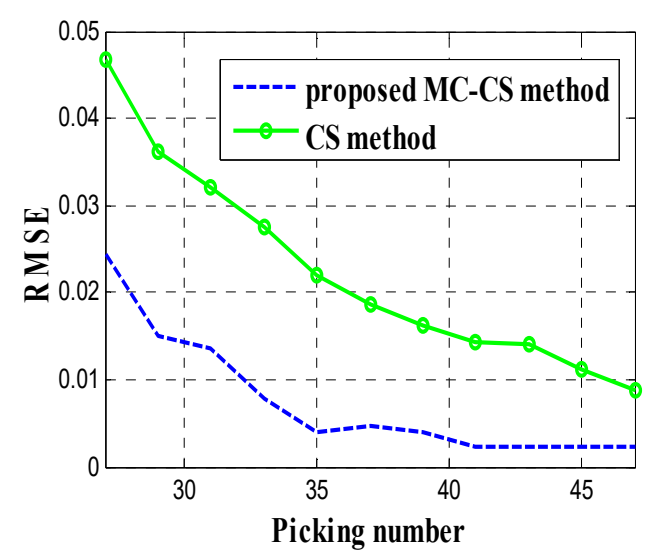

Fig. 4 Average RMSE of DOA estimation with different picking number

\section{Conclusions}

A super resolution DOA estimation with incomplete single snapshot data via matrix completion and compress sensing is proposed in this paper. Numerical simulations verify that the proposed MC-CS method can get super 
resolution estimation performance meanwhile reduce the array number to a certain.

\section{Acknowledgements}

This work was supported by the National Natural Science Foundation of China under grant 61571459, 61372166.

\section{References}

1. B. Radich, K. Buckley,. IEEE SPL, 4, 4(1997)

2. S. Fortunati, R. Grasso, F. Gini, M. Greco, In Proc. 39th IEEE ICASSP, (2014)

3. E. Candes, Y. Plan, Proc. IEEE. 98, 6 (2010).

4. D. Yang, G. Liao, S. Zhu, X. Yang, X. Zhang, IEEE GRSL, 11, 9 (2014).

5. M. Davenport, M. Wakin, IEEE TIT, 56, 9(2010). 